\title{
Editorial
}

\section{Applied Ontology: A foreword by the new Editors-in-Chief}

\author{
Roberta Ferrario $^{\mathrm{a}}$ and Michael Grüninger ${ }^{\mathrm{b}}$ \\ ${ }^{a}$ Laboratory for Applied Ontology, ISTC-CNR, Trento, Italy \\ E-mail: roberta.ferrario@loa.istc.cnr.it \\ ${ }^{\mathrm{b}}$ Department of Mechanical and Industrial Engineering, University of Toronto, Canada \\ E-mail: gruninger@mie.utoronto.ca
}

It might seem odd to speak about the history of a field as young as applied ontology, but given the rapid developments over the past thirty years, it is instructive to consider some of the themes that have motivated research since its inception.

One of the earliest gatherings that focused on applied ontology was the ECAI 1994 Workshop on Implemented Ontologies. Each submission was required to include the specification of the ontologies discussed in the paper, so that discussions about the relationships among ontologies could be based on what was actually being represented rather than on vague unstated assumptions. In 1998, the first international conference on Formal Ontology for Information Systems was held in Trento and applied ontology started to be seen as a unifying discipline having a potential impact on the whole area of information systems, a focus on common scientific principles and its own methodologies, anchored to interdisciplinarity.

In the Proceedings of ECAI $1994^{1}$ and FOIS $1998,{ }^{2}$ we can already see the diversity of domains that are addressed by ontologies. We can also see how the major research questions raised at that time continue to pose challenges to this day. Differences in domain and ontological commitments were exacerbated by the fact that each ontology was represented in its own language; although we have made progress on the standardization of ontology representation languages (such as RDF, OWL, and Common Logic), we cannot say that the problems of ontology shareability, integration, and reuse have been fully solved. Integrating sets of independently designed ontologies and managing the discrepancies among their ontological commitments remain open research problems.

Related to the diversity of the domains in which ontologies are developed is the nature of their application, ranging from semantic annotation and search to semantic integration and automated reasoning. As a journal of applied ontology, it is important to stress how an ontology is actually being used. Each application imposes requirements on an ontology, and even with ontologies in the same domain, one may be adequate for a given application but not for another.

\footnotetext{
${ }^{1}$ Cohn (1994).

${ }^{2}$ Guarino (1998).
} 
We have not yet reached a consensus about ontology evaluation. This is partly the result of the extraordinarily interdisciplinary nature of research within the applied ontology community, with each subfield bringing its own evaluation criteria. Techniques for ontological analysis arise from philosophy, logic, computer science, linguistics, and cognitive science; furthermore, any domain-specific ontology needs to be evaluated with respect to its domain, whether it be biology or systems engineering.

None of these challenges will be solved in this editorial. Nevertheless, we believe that, as a research community, we can perhaps adopt some best-practices that can assist ongoing work:

- make the axiomatization of any ontologies in published papers available in one of the existing ontology repositories (e.g. BioPortal, ${ }^{3}$ OntoHub, ${ }^{4}$ COLORE ${ }^{5}$ );

- be explicit about how the ontologies are being used (e.g. search, semantic integration, decision support), referring to the Ontology Usage Framework from Ontology Summit 2011; ${ }^{6}$

- Make any data used in the application of the ontology publicly available, so that other researchers can reproduce and verify results.

\section{Topics}

Last year, Applied Ontology marked its 10th Anniversary, under the leadership of Nicola Guarino and Mark Musen. We hope to follow in their footsteps and to meet the high standards that they set.

Given the rapid development of many areas within the field of applied ontology, now is a good time to update the scope of the Journal in the light of these advances. Ontological content will remain the emphasis of Applied Ontology Journal, that is to say, ontological analysis and the design and application of specific ontologies to real-world problems rather than the analysis of algorithms, such as the complexity of reasoning in various description logics.

Specialized ontologies. As the name implies, applied ontology has always been driven by applications in a wide-ranging set of domains. It is not possible to completely enumerate all of the relevant domains, but the following list gives some indication of the broad scope of our research community:

- Time, events and processes

- Space and geography

- Physics and physical objects

- Biomedicine

- Mental entities

- Agents and actions

- Organizations and social reality

- Business and e-commerce

- Law

- History, culture and evolution

- Computer Vision

- Design and industry

- Dynamical systems

\footnotetext{
${ }^{3}$ https://bioportal.bioontology.org/

${ }^{4} \mathrm{https}$ ///ontohub.org/

${ }^{5} \mathrm{http}: / /$ stl.mie.utoronto.ca/colore/

${ }^{6}$ http://ontologforum.org/index.php/OntologySummit2011
} 
- Software Engineering

- Socio-Technical Systems and Cyber Physical Systems

- Sensors/Internet of Things Ontologies

- Services

Foundational issues. Underlying the design of ontologies for specific ranges of concepts is the need for solid theoretical foundations based on philosophical, linguistic, and logical analysis.

- Philosophical foundations of ontology

- Basic ontological categories and relations

- Ontology, epistemology, and semiotics

- Impact of ontological analysis on current modelling practices

- Ontological architectures

- Logics for ontology

- Ontology and Knowledge Representation

Ontology management. Ontologies, as theoretical or computational artifacts, can be analyzed through all their life-cycle, from development through their successive versions and comparatively, looking at whether they can be aligned, merged, integrated or made interoperable.

- Methodologies and tools for ontology development, analysis and comparison

- Comparison and evaluation of ontologies

- Ontology management, maintenance, versioning

- Methodologies for ontology merging, alignment, and integration

- Ontology modularization

- Semantic Web Domain Overlap and Vocabularies

- Reasoning with Ontologies

- Ontology Reuse

- Interoperability

- Best-practice examples and case studies

Ontology and language. The relationship between ontology and language is of maximal importance, as it is through the constrains an ontology imposes over the interpretation of the terms used in a language that we try to capture the concepts that we want to express with such a language. This fundamental relationship can be studied focusing on different aspects:

- Ontology and natural-language semantics

- Ontology and lexical resources

- Ontology and terminology

- Ontology learning techniques and their evaluation

- Role of ontology in natural-language systems

Ontology, cognition, perception. The study of the categorization and classification tasks carried out through the use of ontologies has its roots in the study of human cognition, thus it is of paramount importance to inquire the influence of perception and cognition on categorization:

- Conceptual schemas, perceptual invariances and ontological categorization

- Psychological experiments evaluating the cognitive adequacy of ontological categories 
Ontology and content standards. Through the years, ontologies have become an important element in the strategic digital agenda of several governments, businesses, cultural enterprises. The need for unambiguous definitions and for legally binding terminology has increased enormously, opening up the field to official or de facto standards, which have had a huge influence on various communities.

- Library science

- Knowledge organization

- Museums and cultural repositories

- Multimedia content

- Product descriptions

- Process and service descriptions

- Biomedical and other scientific terminologies

- Using ontologies to evaluate standards

Innovative ontology-based applications. Finally, ontologies must face the challenge of the latest scientific and technological developments:

- AI/Hybrid Learning and Ontologies

- Dealing with uncertainty through the use of ontologies

- Ontology and Big Data/Crowdsourcing

- Empirical data management with ontologies

- Ontology and Named Entities Recognition

\section{References}

Cohn, A.G. (Ed.) (1994). Proceedings of the 11th European Conference on Artificial Intelligence (ECAI94). Chichester/London/New York: Wiley.

Guarino, N. (Ed.) (1998). Formal Ontology in Information Systems (Vol. 46: Frontiers in Artificial Intelligence and Applications). Amsterdam: IOS Press. 